\title{
F-18 fluorodeoxyglucose uptake in lymph nodes and sonographic features on endobronchial ultrasonography predict lymph node metastasis in lung cancer patients
}

\author{
Takashi Yamamichi, Masatoshi Kakihana, Yasuyuki Nitta, Wakako Hamanaka, Naohiro Kajiwara, \\ Tatsuo Ohira, Norihiko Ikeda
}

Department of Surgery, Tokyo Medical University, Tokyo, Japan

Contributions: (I) Conception and design: M Kakihana; (II) Administrative support: M Kakihana; (III) Provision of study materials or patients: M Kakihana; (IV) Collection and assembly of data: M Kakihana; (V) Data analysis and interpretation: M Kakihana; (VI) Manuscript writing: All authors; (VII) Final approval of manuscript: All authors.

Correspondence to: Takashi Yamamichi, MD. Department of Surgery, Tokyo Medical University, 6-7-1 Nishishinjuku, Shinjuku-ku, Tokyo 160-0023, Japan. Email: tak13star.winning@gmail.com.

Background: Sonographic findings of lymph nodes on endobronchial ultrasonography (EBUS) images
have been reported to be useful to predict lymph node metastasis (LNM) in lung cancer patients. F-18
fluorodeoxyglucose (FDG) uptake in lymph nodes was also found to be useful. In this study, we aimed to
clarify whether a combination of sonographic features and maximum standardized uptake values of lymph
nodes (LN-SUVmax) is useful for predicting LNM in lung cancer patients.
Methods: From January 2014 to December 2019, a total of 147 lymph nodes from 104 patients with lung
cancer, who underwent preoperative EBUS and FDG-positron emission tomography (PET)/computed
tomography (CT) followed by surgery were retrospectively assesses. The characteristics of the patients, LN-
SUVmax, and sonographic findings of lymph nodes were reviewed. Predictive factors associated with LNM
were identified using the logistic regression model.
Results: The average size of the lymph nodes was 8.55 (range, $3-22$ ) mm and the average LN-SUVmax
was 5.36 (range, $1.79-31.19$ ). The prevalence of nodal metastasis was $26 / 147$ (17.4\%), including 22 in
mediastinal lymph nodes and 4 in hilar lymph nodes. Multivariate analysis demonstrated four independent
predictive factors for LNM; size, round or oval shape, absence of a central hilar structure, and LN-SUVmax.
The optimal cutoff value for lymph node size and LN-SUVmax were 10 mm and 6.00 , respectively. By
combinating of the two modalities, we obtained the results with sensitivity of $76.9 \%$, specificity of $95.1 \%$ and
accuracy of $93.2 \%$.

Conclusions: A combination of sonographic findings and LN-SUVmax showed a higher diagnostic rate of LNM than either modality alone in lung cancer patients.

Keywords: Lymph node metastasis (LNM); endobronchial ultrasonography (EBUS); maximum standardized uptake values of lymph nodes (LN-SUVmax); lung cancer

Submitted May 10, 2020. Accepted for publication Sep 04, 2020.

doi: $10.21037 /$ jtd-20-1888

View this article at: http://dx.doi.org/10.21037/jtd-20-1888

\section{Introduction}

Lung cancer is the most common cause of cancer deaths globally (1). Non-small cell lung cancer accounts for $80 \%$ of all lung cancers, and usually first metastasizes to the hilar and mediastinal lymph nodes. Therefore, accurate preoperative nodal staging is the first and most important step for resectable lung cancer patients. Nodal staging is usually performed by radiological modalities, such 
as computed tomography (CT) and positron emission tomography (PET)/CT (2), which is based on the increased glucose metabolism of malignant cells. The positronemitting radiopharmaceutical F-18 fluorodeoxyglucose (FDG) is the most widely used agent for oncologic PET/ CT imaging. The standardized uptake value (SUV) is a quantified index of FDG uptake. However, in a recent study, the sensitivity of PET/CT in predicting lymph node metastasis (LNM) was 68\%, which has resulted in nodal micrometastases (3) going undetected, and the incidence of LNM in patients with negative nodal uptake on PET/CT was $7-18 \%$ (4). Therefore, FDG-PET/CT alone may not be sufficient for an accurate diagnosis of LNM.

In accordance with the American College of Chest Physicians guidelines on nodal staging, additional lymph node biopsy has become increasingly accepted in patients with a radiologically normal mediastinum to evaluate accurate staging (5). Endobronchial ultrasoundguided transbronchial needle aspiration (EBUS-TBNA) was introduced in 2004 as a less invasive modality for the diagnosis of LNM (6), and is commonly used for pathological preoperative diagnosis. Recently, some researchers have individually reported that sonographic gray features and the vascular pattern grading system may be helpful in predicting LNM during EBUS-TBNA, based on endoscopic ultrasonography $(7,8)$. Wang et al. reported that sonographic features of EBUS with color doppler sonography achieved a favorable diagnostic yield for predicting LNM in lung cancer patients (7). A simplified approach of using at least two of the four predictive factors, which includes round shape, absence of a central hilar structure, presence of matting and nonhilar vascular pattern perfusion may assist in the prediction of LNM. The reason is that metastatic nodes always tend to be larger, round, having no visible hilum (7). In addition, it was reported that matting of the lymph node is a sign of malignancy (7), which suggests the extracapsular spread of malignancy.

In this study, we aimed to identify whether a combination of the measurement of maximum standardized uptake value in lymph nodes (LN-SUVmax) and sonographic features of EBUS-TBNA as proposed by Fujiwara and colleagues (8) is useful in predicting LNM for lung cancer patients, and to determine which factors of these modalities predict LNM accurately.

We present the following article in accordance with the STARD reporting checklist (available at http://dx.doi. org/10.21037/jtd-20-1888).

\section{Methods}

\section{Study population}

A retrospective cohort study was performed on 104 lung cancer patients (147 lymph nodes) who underwent preoperative FDG-PET/CT in a designated facility, followed by preoperative EBUS-TBNA and subsequent pulmonary resection at Tokyo Medical University between January 2014 and December 2019. Patients who underwent EBUS-TBNA within 2 months before surgery were analyzed. In our institution, preoperative EBUSTBNA was performed for patients who has at least one target lymph node larger than $10 \mathrm{~mm}$ or with FDG uptake. EBUS is performed as much as possible to check all lymph nodes. We check the presence of at least three stations of lymph nodes, and TBNA is only performed for the lymph nodes that could affect the surgical procedure or policy. The patients' characteristics, including age, sex, smoking history, histological subtype, and pathological stage were obtained from clinical records. Patients were excluded if (I) preoperative FDG-PET/CT and EBUS-TBNA was not performed; (II) preoperative FDG-PET/CT was performed in other facilities; or (III) SUV was not measurable on a 3D workstation. Lymph node stations and numbers were determined in accordance with the eighth edition of the TNM classification of malignant tumors. Patients were divided into two groups according to their LNM (Figure 1). Lymph nodes with lung cancer or nonspecific inflammation were confirmed on surgical-pathologic confirmation. Lymph nodes were dissected together with the adipose connective tissue of the corresponding anatomic regions, as designated by the surgeon intraoperatively. The study was conducted in accordance with the Declaration of Helsinki (as revised in 2013). The study was being applied by the institutional review board and informed consent was taken from all the patients.

\section{EBUS-TBNA and sonographic findings of lymph nodes}

EBUS-TBNA was performed on patients who had not previously received treatment, who had radiologically defined mediastinal or hilar lymph nodes, and with at least one node of more than $1 \mathrm{~cm}$ in the short-axis on chest CT or with FDG uptake. EBUS-TBNA was performed under local anesthesia with moderate conscious sedation using midazolam. After oral white-light bronchoscopy, the target lesions and peripheral vessels were examined by EBUS, using a linear array ultrasonic bronchoscope (BFUC260F- 


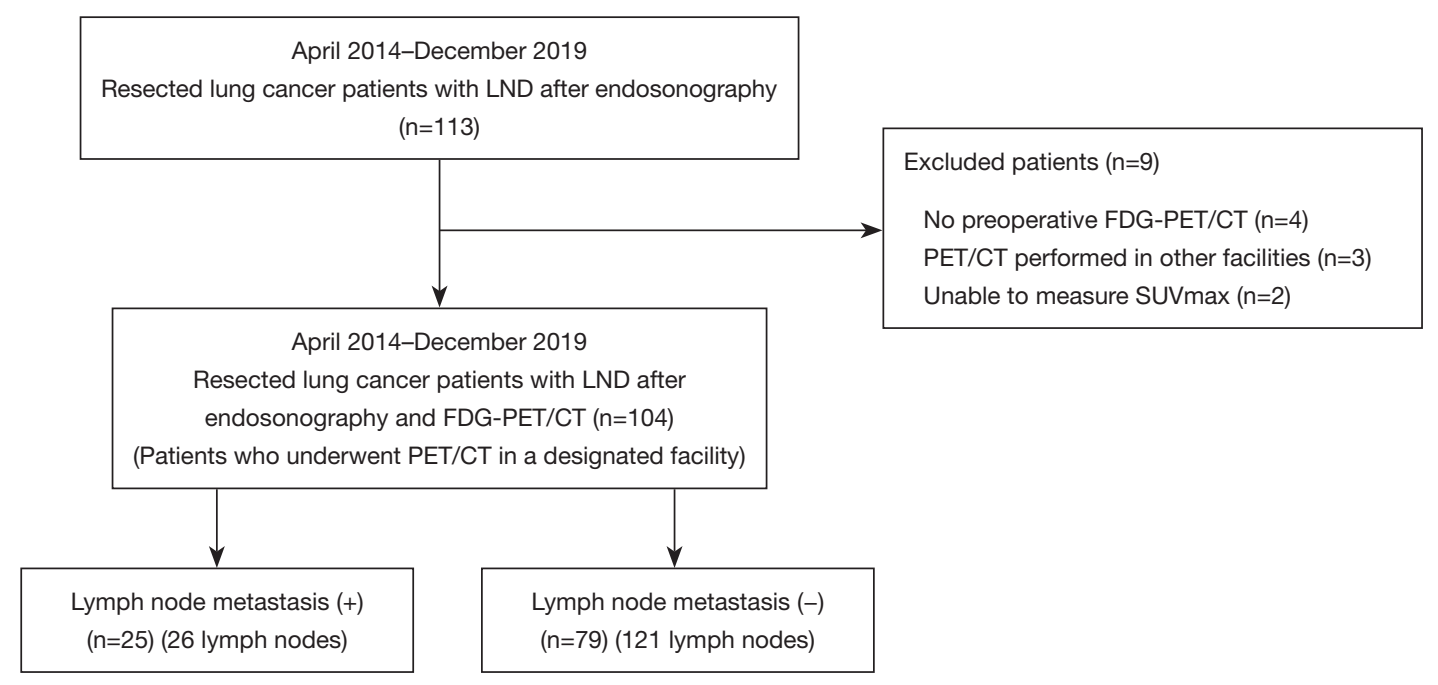

Figure 1 Consort diagram of patients included in the study. LND, lymph node dissection; FDG, fluorodeoxyglucose; PET, positron emission tomography; CT, computed tomography; SUV, standardized uptake value.

OL8; Olympus, Tokyo, Japan). Scanning was performed with a frequency of $7.5 \mathrm{MHz}$, and images were processed by an ultrasound processor (EU-C2000; Olympus). At first, conventional sonographic features were imaged. Final characteristics of the ultrasonography findings for each lymph node were determined, based on the agreement of two sonologists (MK and $\mathrm{WH}$ ). The lymph nodes were characterized based on EBUS sonographic images, including gray scale and power Doppler; the characteristics analyzed were as follows: (I) size, short axis; (II) shape, round or oval; (III) margin, indistinct or distinct; (IV) central hilar structure, presence or absence; (V) echogenicity, homogeneous or heterogeneous; (VI) necrosis signs, present or absent; (VII) calcification, presence or absence; and (VIII) vascular patterns, avascular, hilar perfusion, and nonhilar perfusion. Representative B-mode imaging features are shown in Figure 2.

\section{FDG-PET/CT assessment and measurement of SUVmax of lymph nodes}

All FDG-PET/CT data obtained from the designated facility were used in this study. Patients with blood glucose values of more than $150 \mathrm{mg} / \mathrm{dL}$ during PET/CT imaging were excluded. All patients were assessed using a Biograph PET/CT scanner (Siemens Healthcare, Erlangen, Germany). Unenhanced CT images of sections 2 to $4 \mathrm{~mm}$ thick that matched the PET images were acquired for each patient using a standard protocol. Immediately after the
CT, PET covering the identical axial field of view for 2 to 4 min per table position depending on the condition of the patient and the scanner performance was conducted. SUVmax was established by drawing regions of interests (ROIs) on attenuation-corrected FDG-PET images, and calculated using the software integrated within the PET/ CT scanner based on the following formula:

$$
\text { SUVmax }=M(M B q / k g) /[I D(M B q) / w(k g)]
$$

In which $M$ is defined as the maximal activity at a single pixel within the tissue designated by the ROI, and ID is defined as the injected FDG dose $/ \mathrm{kg}$ of body weight (w).

The preoperative FDG-PET/CT images were transferred to the Synapse Vincent 3D workstation (Fujifilm Corporation, Tokyo, Japan). LN-SUVmax was measured for each patient. ROIs were drawn and maximum FDG uptake in the lymph node was confirmed on CT. Figure 3 shows representative lymph node images of no. 7 lymph node (A), no. 4R lymph node (B), and no. 4L lymph node (C).

\section{Statistical analysis}

Univariate and multivariate logistic regression analyses were performed to identify predictors of LNM using the following sonographic factors: (I) size, (II) shape, (III) margin, (IV) central hilar structure, (V) echogenicity, (VI) necrosis signs, (VII) calcification, (VIII) vascular patterns, and (IX) LN-SUVmax. A backward stepwise selection method was used to build logistic regression 


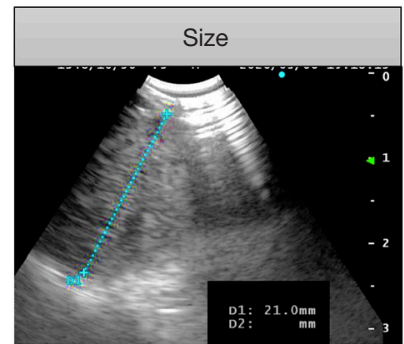

$\geq 10 \mathrm{~mm}$ or not

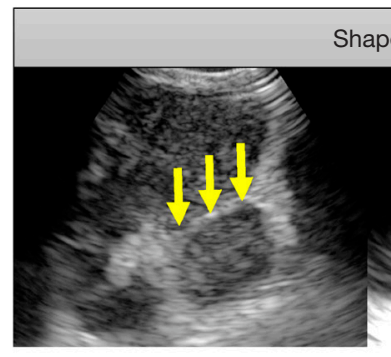

Round

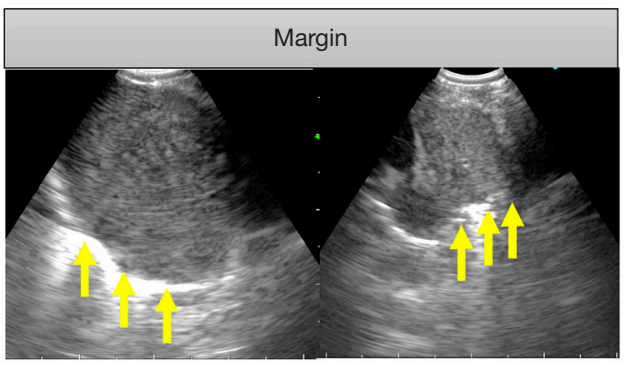

Distinct

Indistinct

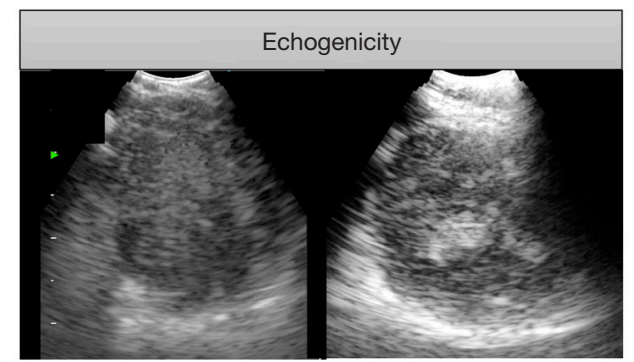

Homogeneous

Heterogeneous

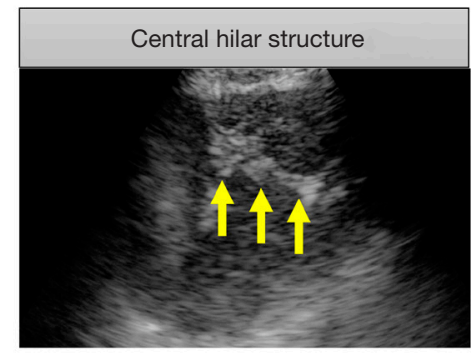

Present or not

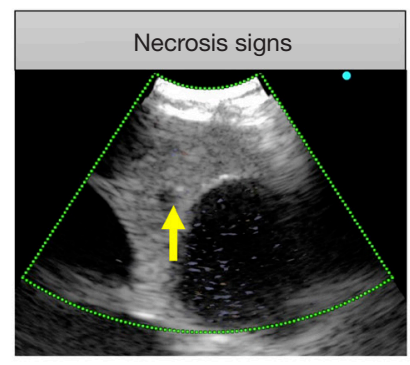

Present or not

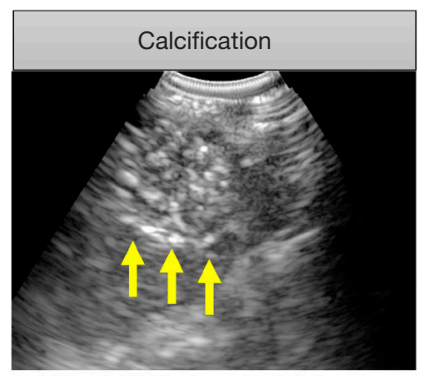

Present or not

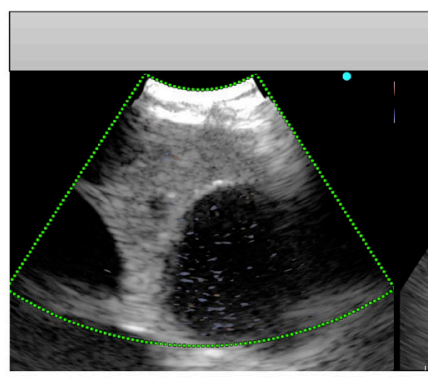

Avascular
Vascular patterns

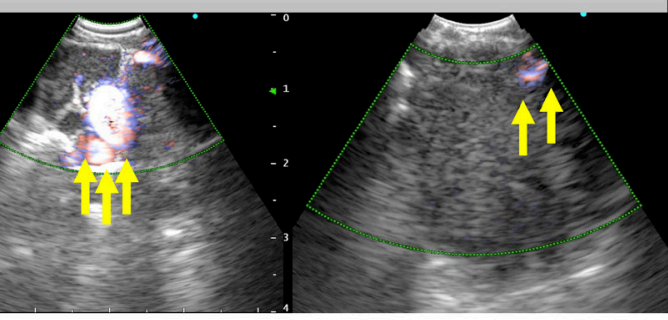

Hilar

Nonhilar

Figure 2 Representative sonographic features of EBUS. Representative images used for classifying metastatic and reactive lymph nodes are shown. Characteristics used were size, $\geq 10 \mathrm{~mm}$ or not; shape, round or oval; central hilar structure, present or absent; margin, distinct or indistinct; echogenicity, homogeneous or heterogeneous; necrosis signs, present or absent; calcification, present or absent; vascular patterns, avascular, hilar, or nonhilar. The arrow is the part that shows the sonographic findings below. EBUS, endobronchial ultrasonography.

models. The receiver operating characteristic (ROC) area under the curve (AUC) of significant factors associated with LNM was measured, in which the cutoff level on the ROC curves for significant parameters was considered to represent a sensitivity/specificity pair corresponding to a particular decision threshold. Categorical comparisons were performed using the Pearson chi-squared test for discrete data and the Mann-Whitney $\mathrm{U}$ test or one-way analysis of variance for continuous data. All tests were two-sided, and $\mathrm{P}$ values less than 0.05 were considered to indicate a statistically significant difference between two groups. The SPSS statistical software package (version 26.0; DDR3 RDIMM, SPSS Inc., Chicago, IL, USA) was used for statistical analyses.

\section{Results}

A total of 104 patients $\{64 \%$ men; median age: 67 [25$86]$ years; $25 \%(26 / 104)$ nonsmokers\} were enrolled in the study. No adverse events were observed. The histological subtypes of primary lung cancer were adenocarcinoma in 68 patients, squamous cell carcinoma in 18 patients, and others in 18 patients. The characteristics of the patients according to LNM are shown in Table 1. The patients who had LNM tended to be young, male, and have advanced stage cancer. EBUS-TBNA was performed in 147 lymph nodes (rate: 1.41 per patient). No severe complications occurred. All pathological findings of EBUS-TBNA were confirmed by subsequent surgery. The prevalence of nodal metastasis was 26/147 (17.4\%), including 22 mediastinal lymph nodes and 

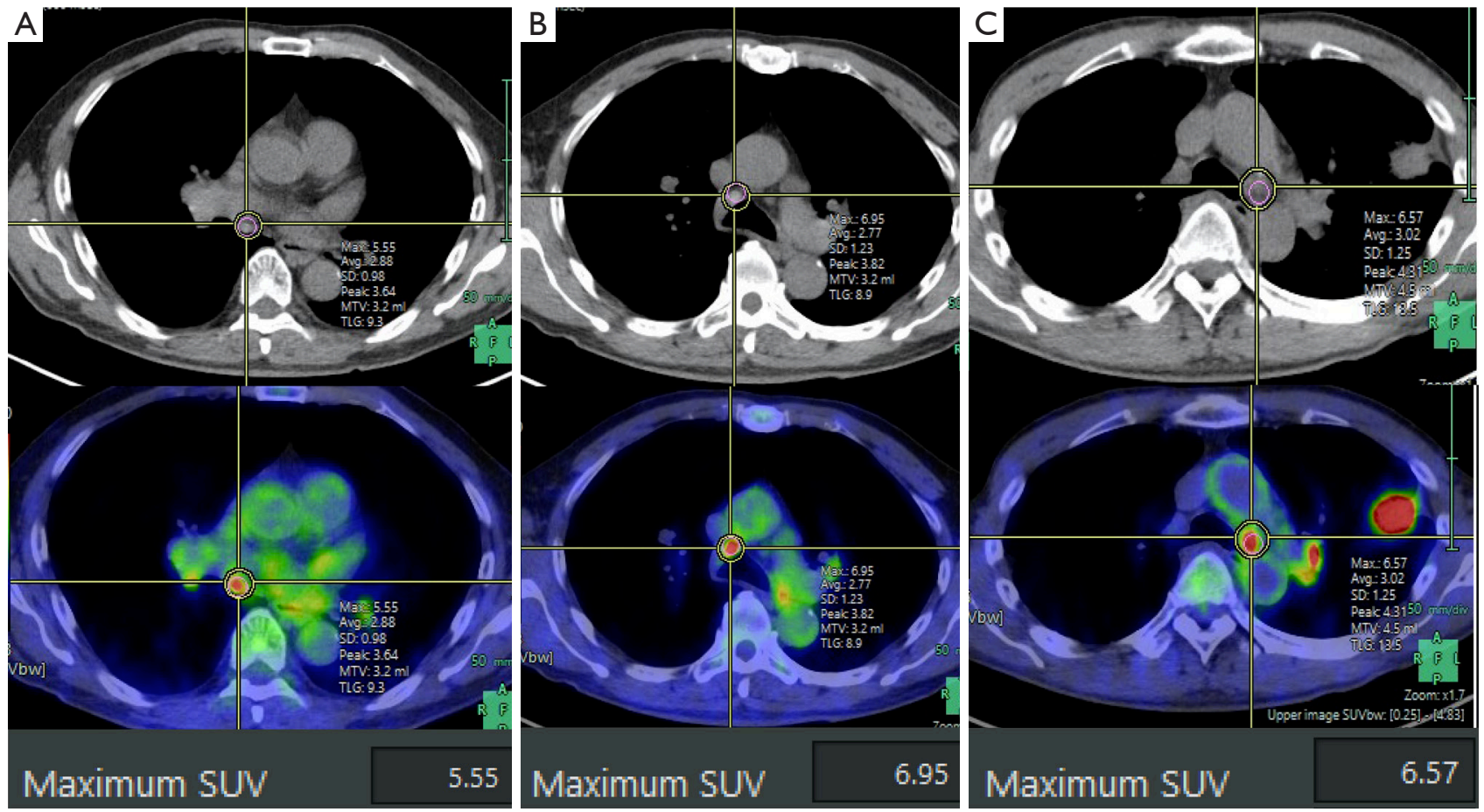

Figure 3 Representative images of the evaluation of LN-SUVmax in noncancerous lung areas obtained using a 3D workstation. (A) Lymph node no. 7; (B) lymph node no. 4R; (C) lymph node no. 4L. LN-SUVmax, maximum standardized uptake value in lymph nodes.

Table 1 Patient characteristics

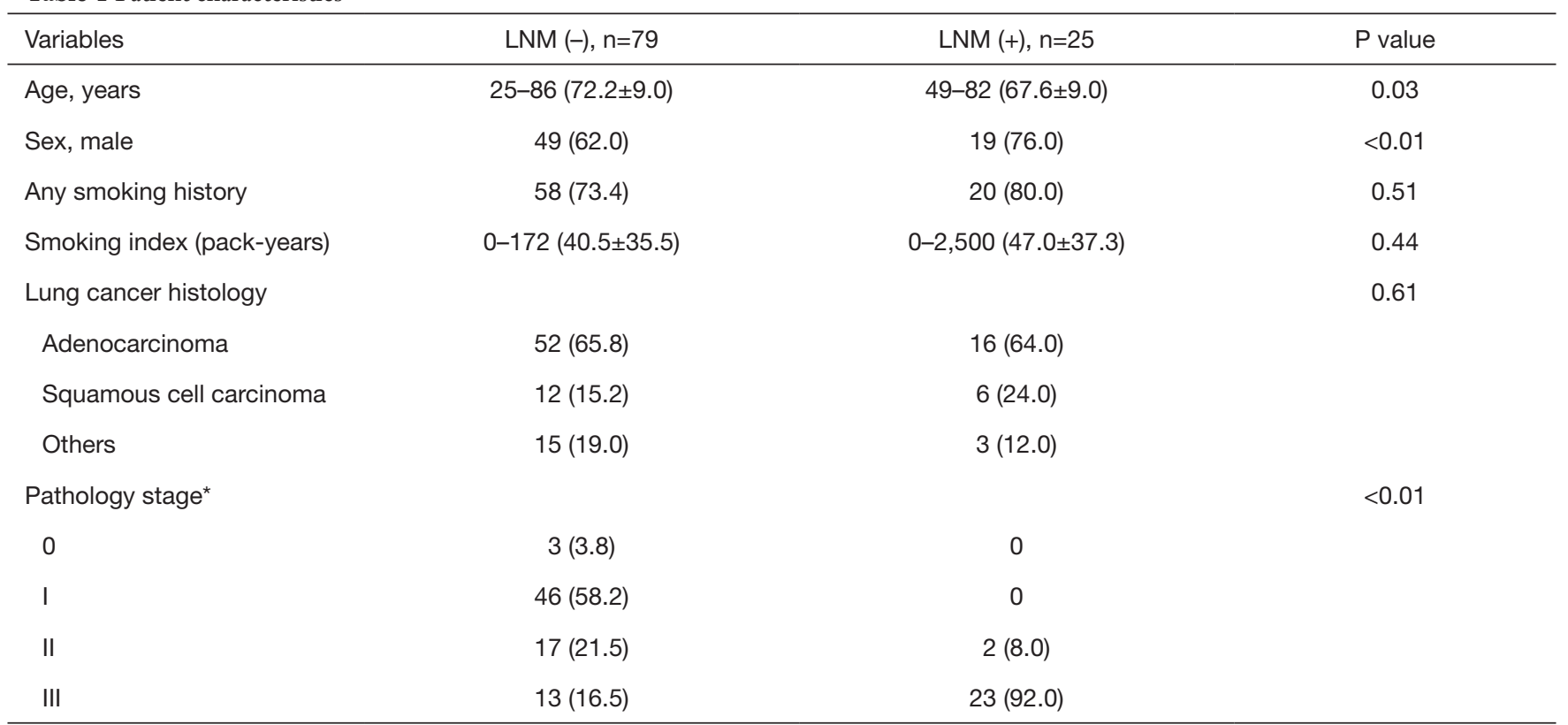

Values are range (average $\pm \mathrm{SD}$ ) or $\mathrm{n}(\%) .{ }^{*}$, based on the eighth edition of the TNM classification of malignant tumors. LNM, lymph node metastasis; SD, standard deviation. 
Table 2 Lymph node characteristics and station according to LNM

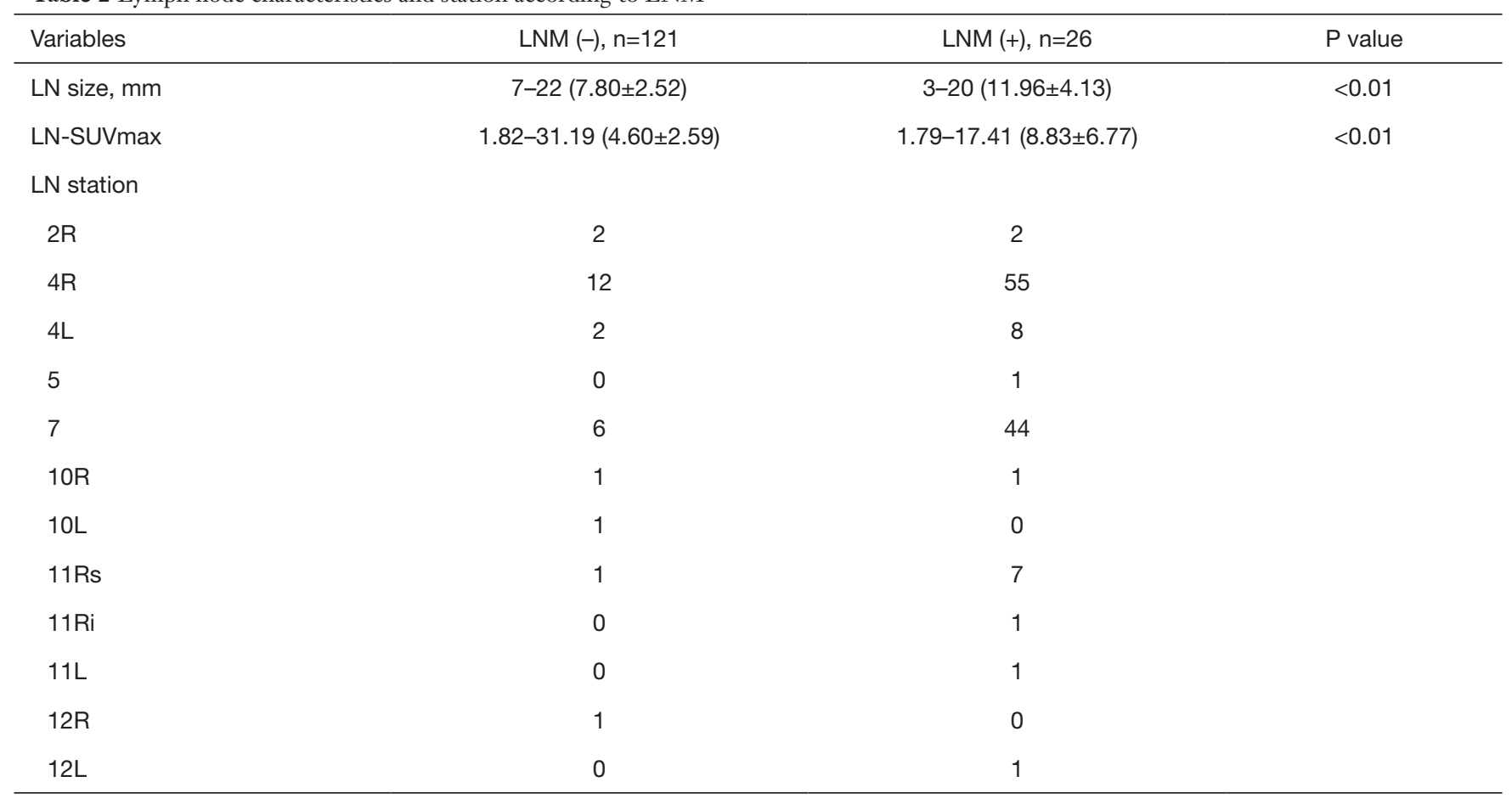

Values are range (average \pm SD). LNM, lymph node metastasis; LN, lymph node; LN-SUVmax, maximum standardized uptake value in lymph nodes; SD, standard deviation.

4 hilar lymph nodes. The average size of the lymph nodes was 8.55 (range, 3-22) $\mathrm{mm}$ and the average LN-SUVmax was 5.36 (range, 1.79-31.19). The characteristics of the lymph nodes are shown in Table 2.

We performed univariate and multivariate analyses to investigate the factors associated with LNM. Size $(\mathrm{P}<0.001)$, round shape $(\mathrm{P}<0.001)$, absence of a central hilar structure $(\mathrm{P}<0.001)$, presence of necrosis signs $(\mathrm{P}<0.001)$, and $\mathrm{LN}-$ SUVmax $(\mathrm{P}<0.001)$ were identified as independent factors on univariate analysis. In addition, size $(\mathrm{P}=0.03)$, round shape $(\mathrm{P}<0.001)$, absence of a central hilar structure $(\mathrm{P}=0.01)$, and LN-SUVmax $(\mathrm{P}=0.02)$ were independent factors on multivariate analysis for LNM (Table 3). We calculated the ROC curves for the incidence of LNM, to investigate the effects of lymph node size and LN-SUVmax. The AUC and the optimal cutoff value of lymph node size relevant to LNM were 0.799 and $10.0 \mathrm{~mm}$, respectively, and those of LN-SUVmax relevant to LNM were 0.747 and 6.00, respectively (Figure 4). To assess whether a combination of criteria would increase the differentiation of malignant and reactive lymph nodes, a model sum score system was investigated. The sensitivity, specificity, and accuracy of endobronchial sonographic features for the prediction of nodal metastasis were $61-92 \%, 41-81 \%$, and $50-78 \%$, respectively. However, the combination of sonographic features and LN-SUVmax showed a higher diagnostic yield than either modality alone (Table 3). According to the highest diagnostic accuracy (93.2\%), the performance of sensitivity, specificity, and positive predictive value (PPV) and negative predictive value (NPV) of lymph nodes having three of the four features (size $>10$ $\mathrm{mm}$, round shape, absence of central hilar structure, LNSUVmax >6.00) for predicting LNM was 76.92\%, 95.12\%, $83.33 \%$, and $96.69 \%$, respectively (Table 4).

\section{Discussion}

Metastasis of intrathoracic lymph nodes can be used as a major prognostic factor in lung cancer, and affects the optimal treatment strategy. Although LNM is generally suspected by the size $(>10 \mathrm{~mm})$ and FDG uptake of lymph nodes on CT or PET/CT imaging $(2,9)$, metastatic lymph nodes sometimes lack FDG uptake or are less than $10 \mathrm{~mm}$. Therefore, in this study, we sought to identify independent risk factors of LNM, including sonographic findings and FDG uptake of lymph nodes. We found that size, shape, 
Table 3 Univariate and multivariate analysis for factors associated with LNM among sonographic features and SUVmax

\begin{tabular}{|c|c|c|c|c|}
\hline Variables & $\begin{array}{l}\text { Univariate analysis, } \\
\text { hazard ratio }(95 \% \mathrm{Cl})\end{array}$ & $P$ value & $\begin{array}{l}\text { Multivariate analysis, } \\
\text { hazard ratio }(95 \% \mathrm{Cl})\end{array}$ & $P$ value \\
\hline \multicolumn{5}{|l|}{ Sonographic features } \\
\hline Size* & $1.50(1.26-1.78)$ & $<0.01$ & $1.26(1.02-1.56)$ & 0.03 \\
\hline Shape: round & $28.33(6.36-126.25)$ & $<0.01$ & $23.21(4.30-125.15)$ & $<0.01$ \\
\hline \multicolumn{5}{|l|}{ Echogenicity: homogeneous } \\
\hline Necrosis signs & $4.05(1.52-10.80)$ & $<0.01$ & & \\
\hline \multicolumn{5}{|l|}{ Vascular pattern: avascular } \\
\hline
\end{tabular}

*, hazard ratio for $1 \mathrm{~mm}$ increment of the size, one increment of LN-SUVmax. LNM, lymph node metastasis; Cl, confidence interval; LNSUVmax, maximum standardized uptake value in lymph nodes.

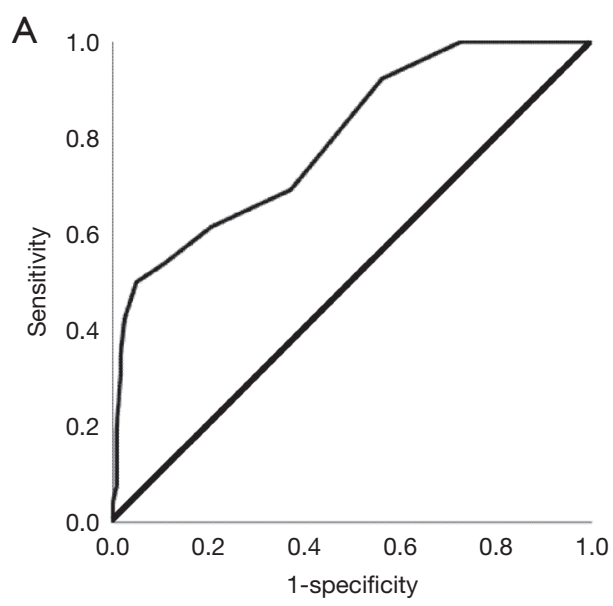

AUC: 0.799 Cutoff level of LN size: $10.0 \mathrm{~mm}$

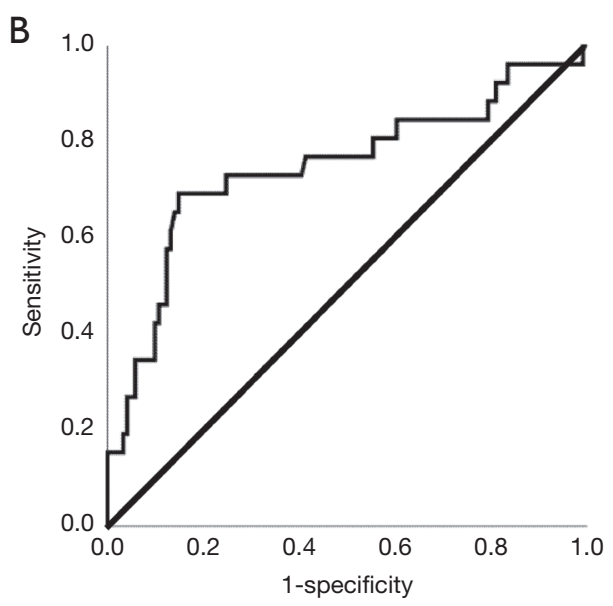

AUC: 0.747 Cutoff level of LN-SUVamx: 6.00

Figure 4 ROC AUCs for lymph node size and LN-SUVmax, used to identify the probability of LNM. ROC AUCs for (A) LN size and (B) LN-SUVmax are shown. ROC, receiver operating characteristic; AUC, area under the curve; LN-SUVmax, maximum standardized uptake value in lymph nodes; LNM, lymph node metastasis; LN, lymph node.

central hilar structure, and LN-SUVmax are useful factors for identifying metastatic and reactive lymph nodes. The AUC and cutoff level associated with LNM for LNSUVmax was 0.747 and 6.00 , respectively, and for lymph node size was 0.799 and $10.0 \mathrm{~mm}$, respectively. Based on multivariate analysis, features with the highest sensitivity for predicting malignancy were the absence of a central hilar structure and round shape; however, these criteria had poor specificity ( $40.5 \%$ and $72.7 \%$, respectively), which was similar to previous reports $(7,8)$. The feature with the highest specificity in this study was a LN-SUVmax of more than 6.00; however, this had poor sensitivity (69.2\%). Hence, the scoring system showed that the presence of at least three of the four independent features in a lymph node [size $(>1 \mathrm{~cm})$, round shape, absence of a central hilar structure, and LN-SUVmax $>6.00$ ] provided the best diagnostic accuracy (93.2\%), which is higher than that of either EBUS or PET/ CT alone. When none of the four features was present, the 
Table 4 Diagnostic performance of endosonography and SUVmax for LNM

\begin{tabular}{|c|c|c|c|c|c|c|}
\hline Variables & Number of patients & Sensitivity, \% & Specificity, \% & PPV, \% & NPV, \% & Accuracy, \% \\
\hline Size $>10 \mathrm{~mm}$ & 39 & 61.5 & 81.0 & 41.0 & 90.7 & 77.5 \\
\hline Shape: round & 57 & 92.3 & 72.7 & 42.1 & 97.8 & 76.2 \\
\hline Central hilar structure: absent & 96 & 92.3 & 40.5 & 25.0 & 96.1 & 49.7 \\
\hline \multicolumn{7}{|l|}{ LN-SUVmax } \\
\hline LN-SUVmax $>6.00$ & 37 & 69.2 & 84.3 & 48.6 & 92.7 & 81.6 \\
\hline \multicolumn{7}{|l|}{ LN-SUVmax + sonographic features } \\
\hline LN-SUVmax $>6.00+$ one feature & 31 & 65.4 & 92.2 & 54.8 & 87.6 & 84.4 \\
\hline LN-SUVmax $>6.00+$ two features & 18 & 65.4 & 99.2 & 94.4 & 93.0 & 93.2 \\
\hline
\end{tabular}

LNM, lymph node metastasis; PPV, positive predictive value; NPV, negative predictive value; LN-SUVmax, maximum standardized uptake value in lymph nodes.

calculated probability of having malignancy was less than $1 \%$.

The National Comprehensive Cancer Network (NCCN) and Yasufuku et al. recommend EBUS-TBNA for the evaluation of mediastinal lymph nodes in patients with lung cancer who are candidates for surgery $(1,10)$. Although EBUS-TBNA has high specificity and a high predictive value for the diagnosis of malignant lymph nodes (11), the diagnostic accuracy of this procedure depends on the accurate selection of the target lymph nodes. Our results have both similarities and differences compared with recent retrospective studies in patients with lung cancer undergoing EBUS $(4,8,12)$. These reports showed that a lymph node size of greater than $1 \mathrm{~cm}$, round shape, heterogeneous echogenicity, distinct margin, and necrosis signs suggest malignancy. We found that size $(>1 \mathrm{~cm})$ and round shape were predictive of malignancy, but that the other characteristics were not. We were unable to identify the other two ultrasound characteristics, i.e., heterogeneous echogenicity and the presence of necrosis signs. One reason may be that this study was a retrospective study and selection bias might have affected the results. However, given that there were several lymph nodes that showed the above sonographic findings but were not found to be malignant, additional factors associated with LNM need to be identified for a more accurate diagnosis.

F-18 FDG-PET/CT is the standard assessment modality for lung cancer patients, with the results greatly affecting patient management, staging, and the prediction of survival outcomes $(13,14)$. Recently, it has been reported that F-18 FDG-PET has great potential for providing additional information (15). FDG-PET/CT is useful for the detection of LNM in clinical practice. The criteria of the cutoff level of SUVmax for predicting LNM was higher than 2.5 (16). However, there are few reports to our knowledge regarding the association between SUVmax and LNM. Our study showed a cutoff level of LN-SUVmax of 6.00, in which the rate of LNM was significantly higher in the LNSUVmax $\geq 6.00$ group. However, this value was obtained from patients in whom a diagnosis of lung cancer had been confirmed and EBUS was performed, and hence the actual value may be slightly lower.

Only one lymph node showed the presence of a central hilar structure, was oval, small (9 mm), and with a LNSUVmax of 4.14. This lymph node was not detected by our criteria. In this case, it was difficult to obtain an adequate diagnosis by sonographic features and LN-SUVmax, and biopsy like EBUS-TBNA or surgery was required to determine the diagnosis. The histological subtype of this lymph node was adenocarcinoma, and cases such as this one are referred to as "occult lymph node metastasis". Hung et al. (17) analyzed factors associated with occult LNM, and found that women, greater tumor size, presence of a micropapillary pattern, and presence of a solid pattern were significant predictors of occult N2 LNM in their model. Miao et al. (18) also showed that the CEA value, solid nodules, SUVmax of the primary tumor, SUVmax of the 
hilar lymph node $\geq 2.0$, and central location are associated with LNM. However, there are no reports to date that occult LNM is associated with sonographic findings. Furthermore, in our present study, it was not possible to detect occult LNM on sonography, and hence we believe that it is important to detect the metastasis of such lymph nodes based on other patient characteristics and imaging modalities, instead of sonographic findings.

This study has several limitations. First, this study was a retrospective case-control study performed at a single institution, and a prospective study is warranted to confirm our findings. Second, selection bias might have affected the results. We excluded patients who underwent chemotherapy or chemoradiotherapy before surgery, because we wanted to analyze patients with resectable and marginal zone tumors. Third, subjective interpretation of the EBUS images was inevitable. For example, homogenous and margin distinct are particularly likely to be objective evaluations. To reduce these bias, the final characteristics of the EBUS findings were determined based on the agreement of two sonologists to whom the pathology results were not disclosed. Fourth, this study was targeted at the lung cancer patients who underwent preoperative EBUS-TBNA and surgery, and not all patients who underwent EBUS-TBNA in our hospital. As a result, the number of lymph nodes evaluated is less than in previous reports.

In conclusion, a combination of sonographic findings and LN-SUVmax resulted in a higher diagnostic rate of LNM than either modality alone. This knowledge is expected to assist clinicians in identifying the most suspicious lymph nodes for puncturing during EBUS-TBNA, which may improve the efficiency of EBUS-TBNA procedures.

\section{Acknowledgments}

The authors are indebted to the medical editors from the Department of International Medical Communications of Tokyo Medical University, for editing of the English manuscript.

Funding: None.

\section{Footnote}

Reporting Checklist: The authors have completed the STARD reporting checklist. Available at http://dx.doi. org/10.21037/jtd-20-1888

Data Sharing Statement: Available at http://dx.doi. org/10.21037/jtd-20-1888

Peer Review File: Available at http://dx.doi.org/10.21037/jtd20-1888

Conflicts of Interest: All authors have completed the ICMJE uniform disclosure form (available at http://dx.doi. org/10.21037/jtd-20-1888). The authors have no conflicts of interest to declare.

Ethical statement: The authors are accountable for all aspects of the work in ensuring that questions related to the accuracy or integrity of any part of the work are appropriately investigated and resolved. The study was conducted in accordance with the Declaration of Helsinki (as revised in 2013). The study was being applied by the institutional review board and informed consent was taken from all the patients.

Open Access Statement: This is an Open Access article distributed in accordance with the Creative Commons Attribution-NonCommercial-NoDerivs 4.0 International License (CC BY-NC-ND 4.0), which permits the noncommercial replication and distribution of the article with the strict proviso that no changes or edits are made and the original work is properly cited (including links to both the formal publication through the relevant DOI and the license). See: https://creativecommons.org/licenses/by-nc-nd/4.0/.

\section{References}

1. Ettinger DS, Wood DE, Akerley W, et al. Non-small cell lung cancer, version 6.2015. J Natl Compr Canc Netw 2015;13:515-24.

2. Billé A, Pelosi E, Skanjeti A, et al. Preoperative intrathoracic lymph node staging in patients with nonsmall-cell lung cancer: accuracy of integrated positron emission tomography and computed tomography. Eur J Cardiothorac Surg 2009;36:440-5.

3. Song CY, Kimura D, Sakai T, et al. Novel approach for predicting occult lymph node metastasis in peripheral clinical stage I lung adenocarcinoma. J Thorac Dis 2019;11:1410-20.

4. Lv YL, Yuan DM, Wang K, et al. Diagnostic performance of integrated positron emission tomography/computed tomography for mediastinal lymph node staging in nonsmall cell lung cancer: a bivariate systematic review and meta-analysis. J Thorac Oncol 2011;6:1350-8. 
5. Silvestri GA, Gonzalez AV, Jantz MA, et al. Methods for staging non-small cell lung cancer: diagnosis and management of lung cancer, 3rd ed: American College of Chest Physicians evidence-based clinical practice guidelines. Chest 2013;143:e211S-50S.

6. Yasufuku K, Chiyo M, Sekine Y, et al. Real-time endobronchial ultrasound-guided transbronchial needle aspiration of mediastinal and hilar lymph nodes. Chest 2004;126:122-8.

7. Wang $\mathrm{L}, \mathrm{Wu} \mathrm{W}, \mathrm{Hu} \mathrm{Y}$, et al. Sonographic features of endobronchial ultrasonography predict intrathoracic lymph node metastasis in lung cancer patients. Ann Thorac Surg 2015;100:1203-9.

8. Fujiwara T, Yasufuku K, Nakajima T, et al. The utility of sonographic features during endobronchial ultrasoundguided transbronchial needle aspiration for lymph node staging in patients with lung cancer: a standard endobronchial ultrasound image classification system. Chest 2010;138:641-7.

9. Glazer GM, Gross BH, Quint LE, et al. Normal mediastinal lymph nodes: number and size according to American Thoracic Society mapping. AJR Am J Roentgenol 1985;144:261-5.

10. Yasufuku K, Pierre A, Darling G, et al. A prospective controlled trial of endobronchial ultrasound-guided transbronchial needle aspiration compared with mediastinoscopy for mediastinal lymph node staging of lung cancer. J Thorac Cardiovasc Surg 2011;142:1393400.e1.

11. Khazai L, Kundu UR, Jacob B, et al. Endobronchial ultrasound-guided transbronchial needle aspiration biopsy is useful evaluating mediastinal lymphadenopathy in a cancer center. Cytojournal 2011;8:10.

Cite this article as: Yamamichi T, Kakihana M, Nitta Y, Hamanaka W, Kajiwara N, Ohira T, Ikeda N. F-18 fluorodeoxyglucose uptake in lymph nodes and sonographic features on endobronchial ultrasonography predict lymph node metastasis in lung cancer patients. J Thorac Dis 2020;12(10):54205429. doi: $10.21037 /$ jtd-20-1888
12. Jenssen C, Annema JT, Clementsen P, et al. Ultrasound techniques in the evaluation of the mediastinum, part 2 : mediastinal lymph node anatomy and diagnostic reach of ultrasound techniques, clinical work up of neoplastic and inflammatory mediastinal lymphadenopathy using ultrasound techniques and how to learn mediastinal endosonography. J Thorac Dis 2015;7:E439-58.

13. Furumoto H, Shimada Y, Imai K, et al. Prognostic impact of the integration of volumetric quantification of the solid part of the tumor on 3DCT and FDG-PET imaging in clinical stage IA adenocarcinoma of the lung. Lung Cancer 2018;121:91-6.

14. Kaira K, Serizawa M, Koh Y, et al. Biological significance of 18F-FDG uptake on PET in patients with non-smallcell lung cancer. Lung Cancer 2014;83:197-204.

15. Yamamichi T, Shimada $Y$, Masuno R, et al. Association between F-18 fluorodeoxyglucose uptake of noncancerous lung area and acute exacerbation of interstitial pneumonia in patients with lung cancer after resection. J Thorac Cardiovasc Surg 2020;159:1111-8.e2.

16. Koksal D, Demirag F, Bayiz H, et al. The correlation of SUVmax with pathological characteristics of primary tumor and the value of tumor/lymph node SUVmax ratio for predicting metastasis to lymph nodes in resected NSCLC patients. J Cardiothorac Surg 2013;8:63.

17. Hung JJ, Yeh YC, Jeng WJ, et al. Factors predicting occult lymph node metastasis in completely resected lung adenocarcinoma of $3 \mathrm{~cm}$ or smaller. Eur J Cardiothorac Surg 2016;50:329-36.

18. Miao H, Shaolei L, Nan L, et al. Occult mediastinal lymph node metastasis in FDG-PET/CT node-negative lung adenocarcinoma patients: risk factors and histopathological study. Thorac Cancer 2019;10:1453-60. 